\title{
Elevated expression of podoplanin and its clinicopathological, prognostic, and therapeutic values in squamous non-small cell lung cancer
}

This article was published in the following Dove Press journal: Cancer Management and Research

\author{
Liyi Xie ${ }^{1-3}$ \\ Chen $\operatorname{Lin}^{4}$ \\ Qingfu Zhang ${ }^{5}$ \\ Hailan Piao ${ }^{6}$ \\ Darell D Bigner ${ }^{3,7}$ \\ Zhen Zhang ${ }^{1,2}$ \\ Xuhui Bao ${ }^{8}$
}

'Department of Radiation Oncology, Fudan University Shanghai Cancer Center, Shanghai, China; ${ }^{2}$ Department of Oncology, Shanghai Medical

College, Fudan University, Shanghai, China; ${ }^{3}$ Department of Neurosurgery, Duke University Medical Center, Durham, NC, USA; ${ }^{4}$ Department of Medical Oncology, Zhejiang Cancer Hospital, Hangzhou, Zhejiang, China; ${ }^{5}$ Department of Pathology, the First Affiliated Hospital and College of Basic Medical Sciences, China Medical University, Shenyang, Liaoning, China; ${ }^{6}$ Department of Medicine, Duke University Medical Center, Durham, NC, USA; 'Department of Pathology, Duke University Medical Center, Durham, NC, USA; ${ }^{8}$ Department of Surgery, Duke University Medical Center, Durham, NC, USA

Correspondence: Zhen Zhang; Liyi Xie Department of Radiation Oncology, Fudan University Shanghai Cancer Center, 270 Dong An Rd, Shanghai 200032, China

Tel +862164175590

Fax +86 21 64174774

Email zhenzhang6@gmail.com; xie.li.yi@ duke.edu
Background: Squamous non-small cell lung cancer (SqNSCLC), as a leading cause of cancerrelated deaths worldwide, has limited treatment options and poor prognosis. Thus, novel targeted therapies are desperately needed.

Materials and methods: SqNSCLC cases from derivation and validation cohorts were analyzed for podoplanin (PDPN) expression, and its clinicopathological correlation and prognostic prediction. The Human Proteome Map database was used to compare the expression of different lung cancer targets in normal human tissues. Two human lung cancer cell lines, H226 (a SqNSCLC line) and A549 (a non-SqNSCLC line), were examined for PDPN expression. The in vitro cytotoxicity of an anti-PDPN therapy (NZ-1-immunotoxin [NZ-1-IT]) was tested against both lines. The in vivo therapeutic effect of NZ-1-IT was examined in subcutaneous non-small cell lung cancer (NSCLC) xenograft mouse models.

Results: In the derivation cohort, 40\% (28/70) were PDPN positive. There was significantly increasing pleural invasion ( $46.4 \%$ vs $9.5 \%, p=0.001)$, lymphovascular invasion $(25.0 \%$ vs $9.5 \%, p=0.08)$, and lymph node involvement (53.6\% vs 33.3\%, $p=0.09$ ) in PDPN-positive vs PDPN-negative patients, along with poorer progression-free survival in PDPN-positive patients $(p=0.07)$. The validation cohort with 224 randomly matched cases from The Cancer Genome Atlas data set also displayed significantly shorter overall survival in the group with elevated $P D P N$ mRNA ( $p=0.05)$. However, PDPN showed limited expression in normal tissues. PDPN was highly and specifically expressed on the surface of H226 cells instead of A549 cells. Subsequently, PDPN-positive H226 cells were around 800 times more sensitive to anti-PDPN NZ-1-IT therapy than PDPN-negative A549 cells in vitro. Furthermore, NZ-1-IT significantly delayed tumorigenesis only in the H226 subcutaneous mouse model $(p<0.05)$.

Conclusion: Our results demonstrate a distinctively elevated expression of PDPN in SqNSCLC, which is significantly associated with worse clinicopathological features and poorer prognosis. With promising preclinical therapeutic results, anti-PDPN targeted therapy can thus be a robust potential strategy for future SqNSCLC treatment.

Keywords: podoplanin, squamous non-small cell lung cancer, pleural invasion, prognosis, molecular targeted therapy, immunotoxins

\section{Introduction}

Lung cancer is the leading cause of cancer-related deaths worldwide, accounting for 1.69 million deaths in 2015 according to the World Health Organization. ${ }^{37}$ In China, lung cancer is also the most common cancer, with an incidence of 730,000 and deaths of 610,000. ${ }^{1}$ Squamous non-small cell lung cancer (SqNSCLC) accounts for one-third of non-small cell lung cancer (NSCLC)., ${ }^{2,3}$ Given that there are more than 300 million 
smokers in China, ${ }^{38}$ along with the fact that SqNSCLC is closely related to smoking in a dose-dependent manner, $2,4,5$ a persistent large patient volume who suffer from voluntary and involuntary smoking-induced SqNSCLC is foreseeable. To date, around $80 \%$ of SqNSCLC patients are diagnosed as advanced stage, with a median survival of about 12 months, and a 5-year survival rate of $28.9 \%$ for regional disease and $4.5 \%$ for distant disease, despite therapeutic progress achieved in the past few decades. ${ }^{2,6,7}$ Even though many advances have been made in understanding the molecular genetics of NSCLC, there are still limited treatment options available for SqNSCLC, and more novel and targeted therapeutics with effective and unique functions are desperately needed for SqNSCLC treatment.

For novel treatment strategies, crossover expression of some therapeutic targets has disrupted the fragile balance between cancer cell killing and normal tissue protection and has been a major impediment. ${ }^{8}$ Throughout the last decade, advances have been made to seek more specific and reliable targets for SqNSCLC. ${ }^{3,9}$ Such advances, focusing on identifying unique molecular abnormalities specifically associated with squamous cell lung cancer but minimally expressed or absent in normal tissue, are vital in the development of more effective SqNSCLC therapeutics. ${ }^{3,9}$ Podoplanin (PDPN) is a 162-amino acid type I transmembrane sialomucin-like glycoprotein with a serine- and threonine-rich extracellular domain, a single transmembrane domain, and a short cytoplasmic domain containing cyclic adenosine monophosphate (cAMP) and protein kinase $\mathrm{C}$ phosphorylation sites. PDPN binds to C-type lectin-like receptor 2 (CLEC2) expressed on platelets and causes platelets to aggregate and promote tumor metastasis. ${ }^{10}$ In addition, PDPN can form a complex with ezrin-radixin-moesin (ERM) protein to increase cell motility by activating RhoA signaling. ${ }^{11}$ Therefore, PDPN is related to malignant progression involving epithelialmesenchymal transition (EMT), metastasis, and invasion in several cancers, ${ }^{11-13}$ which can be a potential therapeutic target for PDPN-expressing malignant tumors. Our previous study revealed a robust antitumor efficacy in a preclinical brain tumor model using an anti-PDPN NZ-1 immunotoxin (NZ-1-IT), ${ }^{14}$ which is developed by fusing a recombinant single-chain variable-region antibody fragment ( $\mathrm{scFv}$ ) from a monoclonal antibody (mAb) with a bacterial toxin. As important derivatives of antibody-drug conjugates (ADCs), $\mathrm{scFv}$-based immunotoxins provide robust tumor penetration, specific tumor binding, and unique tumor killing. These immunotoxins continue to be extensively investigated to treat many cancers, including lung cancer, malignant mesothelioma, pancreatic cancer, gastric cancer, and brain cancer. $^{15-18}$

In the present study, we elucidated the robust clinicopathological, prognostic, and therapeutic value of PDPN in SqNSCLC. We investigated the PDPN expression and the correlation between elevated PDPN expression and pleural invasion, lymphovascular invasion (LVI), lymph node metastases, and patient survival. Subsequently, we demonstrated promising preclinical results of an anti-PDPN immunotoxin therapy in in vitro NSCLC models and in vivo xenograft mouse models.

\section{Materials and methods Study population}

In total, 440 consecutive patients with a pathological diagnosis of single primary NSCLC who underwent curativeintent thoracic surgery in Fudan University Shanghai Cancer Center (FUSCC) from January 2012 to December 2012 were identified, including 101 patients with SqNSCLC. Among them, tissue samples from 70 SqNSCLC patients with complete pivotal clinical information were assessed, and these patients comprised the derivation cohort (Figure 1). Patients were $>20$ years old and newly diagnosed with the American Joint Committee on Cancer (AJCC), 7th edition, stage I-IV SqNSCLC confirmed by surgical pathology. The research was carried out according to the Code of Ethics of the World Medical Association (Declaration of Helsinki). All clinical demographic characteristics and specimens were collected from patients who had provided written informed consent for FUSCC institutional review board (IRB)-approved clinical and laboratory research protocols. Patient follow-up information was obtained from an electronic medical record (EMR) system in FUSCC.

The validation cohort consisted of 504 SqNSCLC patients with known clinical information and genomic data recorded in The Cancer Genome Atlas (TCGA) data sets. PDPN mRNA expression was analyzed to identify patients with high expression of $P D P N$ (above the 75th percentile) and low expression of $P D P N$ (below the 25 th percentile). Randomized matching of the high-expression and low-expression groups was carried out with a 1:1 ratio in terms of AJCC pathological staging. Gene expression data of these two groups were analyzed with cBioPortal tools $;{ }^{39}$ users are free to use any of the figures from the portal in their publications or presentations). ${ }^{19,20}$ TCGA is a public data set that contains deidentified patient-related clinical and genomic information; thus, no IRB or Health Insurance Portability and Accountability Act approval was required for the validation cohort. 
A

FUSCC derivation cohort

440 Index NSCLC cases including 101 SqNSCLC:

1. Single primary SqNSCLC during 2012 (pathological

diagnosis)

2. Curative-intent thoracic surgery

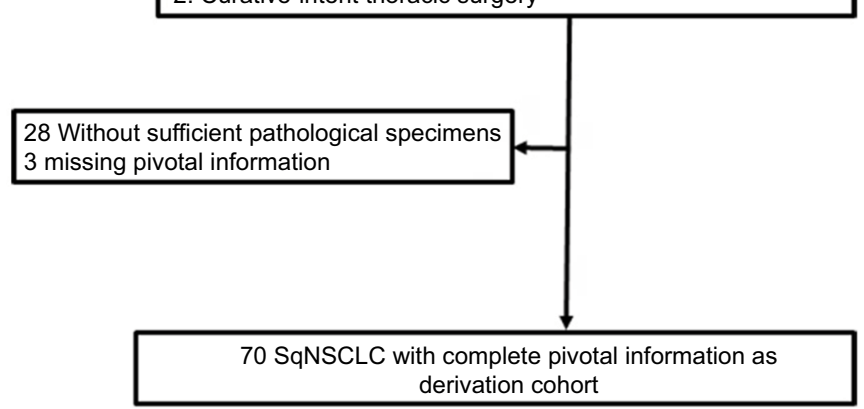

B

TCGA validation cohort

Figure I Study profile to show how samples from (A) FUSCC and (B) TCGA were analyzed.

Abbreviations: FUSCC, Fudan University Shanghai Cancer Center; TCGA, The Cancer Genome Atlas; NSCLC, non-small cell lung cancer; SqNSCLC, squamous non-small cell lung cancer; PDPN, podoplanin; AJCC, American Joint Committee on Cancer.

\section{Normal tissue protein expression data set}

Protein expression data in normal tissues were extracted from the Human Proteome Map (HPM) portal. ${ }^{21,40}$ As part of the HPM project, 30 clinically defined normal tissues were profiled in total, of which 17 were adult human tissues and 6 were primary hematopoietic cells. The project detected proteins corresponding to over 17,000 human genes covering $>84 \%$ of the annotated protein-coding regions of the human genome.

\section{Tissue sample}

Surgical tissue specimens from those 70 patients with SqNSCLC who underwent surgical treatment at FUSCC in 2012 were retrospectively collected following IRB guidelines. Representative tissue sections were obtained from the surgical pathology archive. Paraffin-embedded blocks and corresponding pathological diagnoses were confirmed by the EMR at FUSCC.

\section{Immunohistochemistry}

In brief, the formalin-fixed paraffin-embedded sections were deparaffinized and then heated in $0.01 \mathrm{M}$ citrate buffer $(\mathrm{pH}$ 6.0). Endogenous peroxidase activities were inactivated in $3 \%$ hydrogen peroxide $\left(\mathrm{H}_{2} \mathrm{O}_{2}\right)$, and the sections were blocked with 3\% normal goat serum in PBS (pH 7.4). Sections were incubated with an anti-PDPN antibody (Sigma-Aldrich, St. Louis, MO, USA) and then a secondary goat anti-rat antibody (Sigma-Aldrich), followed by treatment with substrate/ chromogen (DAKO, Santa Clara, CA, USA). Slides were counterstained with hematoxylin. All slides with a confirmed pathological diagnosis from the EMR in FUSCC were independently re-reviewed by a senior surgical pathologist from a different institution (Q. Zhang).

\section{Cell lines}

The A549 cell line was bought from the Cell Bank of Type Culture Collection of Chinese Academy of Sciences, which was cultured in F12K medium (Thermo Fisher Scientific, Waltham, MA, USA) with $10 \%$ fetal bovine serum (FBS; Thermo Fisher Scientific). The H226 cell line was a generous gift from one of our collaborators (D.D. Bigner), which was cultured in RPMI-1640 medium (Thermo Fisher Scientific) modified to contain $2 \mathrm{mM}$ L-glutamine, $10 \mathrm{mM}$ HEPES (Thermo Fisher Scientific), $1 \mathrm{mM}$ sodium pyruvate (Thermo Fisher Scientific), $4500 \mathrm{mg} / \mathrm{L}$ glucose (Sigma-Aldrich, St. Louis, MO, USA), $1500 \mathrm{mg} / \mathrm{L}$ sodium bicarbonate, and 10\% FBS. Both cell lines were maintained and passed according to the American Type Culture Collection (ATCC) recommendations. ${ }^{41}$ The use of the $\mathrm{H} 226$ cell line was approved by FUSCC-IRB.

\section{Flow cytometry analysis}

Flow cytometry analysis was carried out with AF488-labeled (Pierce, Thermo Fisher Scientific, Waltham, MA, USA) NZ-1 mAb (NZ-1-AF488), while rat immunoglobulin G2a (IgG2a)-AF488 isotype control (Thermo Fisher Scientific) was used as the negative control. The fluorescence-labeled antibodies stained $10^{6}$ cells at a concentration recommended by the manufacturer. Stained cells were analyzed on a BD FACSCalibur cytometer (BD Biosciences, San Jose, CA, USA). Data were analyzed using FlowJo V10 software (FlowJo, Ashland, OR, USA). 


\section{Western blot analysis}

Protein samples were extracted from A549 and H226 cells using the Mammalian Protein Extraction Reagent (Thermo Fisher Scientific) and protease inhibitor cocktail (Roche, Basel, Switzerland). After separation and transfer onto the membrane, protein was probed with antibodies against PDPN (1:1000; Sigma-Aldrich) or glyceraldehyde 3-phosphate dehydrogenase (GAPDH, 1:5000; Santa Cruz Biotechnology, Dallas, TX, USA). Proteins of interest were detected with horseradish peroxidase-conjugated goat anti-rat IgG antibody (1:5000; Sigma-Aldrich) and visualized with the Pierce ECL Western blotting substrate (Thermo Fisher Scientific), according to the manufacturer's protocols.

\section{In vitro cytotoxicity assay}

Cells were seeded in 96-well plates at a density of $10^{4}$ cells per well in $100 \mu \mathrm{L}$ of complete medium overnight before the assay. NZ-1-IT was serially diluted to achieve a final concentration of $0.001-1000 \mathrm{ng} / \mathrm{mL}$ in PBS (Thermo Fisher Scientific) containing $0.2 \%$ human serum albumin $(0.2 \%$ HSA-PBS; Shanghai RAAS Blood Products Co., Shanghai, China). Plates were incubated for $48 \mathrm{~h}$ at $37^{\circ} \mathrm{C}$ and $10 \mu \mathrm{L}$ WST-1 reagent (Roche) was then added for $0.5-4 \mathrm{~h}$ at $37^{\circ} \mathrm{C}$. The absorbance of each well was measured against a blank background control at $420-480 \mathrm{~nm}$ wavelength. The reference wavelength was $650 \mathrm{~nm}$. The cytotoxic activity of NZ1-IT was defined by the $\mathrm{IC}_{50}$, which was the immunotoxin concentration that inhibited cell proliferation by $50 \%$ when compared to that of untreated cells. The $\mathrm{IC}_{50}$ was calculated using GraphPad Prism 6 (GraphPad Software, La Jolla, CA, USA). All the assays were performed twice in triplicate for each cell line.

\section{In vivo subcutaneous xenograft NSCLC model}

Animal experiments were approved by the Institutional Animal Care and Use Committee of Fudan University. Female athymic nude mice (7-10 weeks old) were injected subcutaneously in the right flank with $3 \times 10^{6}$ A549 or H226 cells suspended in $100 \mu \mathrm{L}$ of PBS. When the implanted tumors reached around $100 \mathrm{~mm}^{3}$ on day 35 after the implantation, five mice per arm were randomized for treatment. The test mice were treated by intratumoral injection every other day with a total of three doses ( $300 \mu \mathrm{g} / \mathrm{kg}$ per dose) of NZ-1-IT diluted in $0.2 \%$ HSA-PBS, or $0.2 \%$ HSA-PBS as the vehicle control. Tumor growth was monitored in the following 30 days, and the tumor volumes were calculated in cubic millimeters using the formula: $\left([\right.$ Length $] \times\left[\right.$ Width $\left.\left.^{2}\right]\right) / 2$. Tumor growth plots were drawn using GraphPad Prism 6.

\section{Statistical methods}

The pleural invasion, LVI, and lymph node status between PDPN-positive and PDPN-negative cases were compared with the chi-squared test and Fisher's exact test. Overall survival (OS) and progression-free survival (PFS) were defined, respectively, as the time from initial diagnosis to death from any cause, and to local, regional, or distant progression or death from any cause. The distributions of OS and PFS were estimated by the Kaplan-Meier method ${ }^{22}$ and compared to the log-rank test. ${ }^{23} \mathrm{HRs}$ were estimated according to the Cox proportional hazard method. ${ }^{24}$ The statistical analyses for clinical data were conducted using PASW Statistics version 18.0 (SPSS, Chicago, IL, USA). The nonparametric Wilcoxon each-pair multiple comparison method was used to analyze the data from the xenograft lung cancer mouse model using JMP Pro 13.0 (SAS Institute, Cary, NC, USA). Difference was considered significant at $p<0.05$.

\section{Results}

\section{Elevated PDPN expression had a significant impact on patient clinicopathological features and prognosis in SqNSCLC}

The baseline characteristics of the derivation and validation cohorts are listed in Tables 1 and 2. Among the derivation

Table I Clinicopathological parameters of SqNSCLC patients in the derivation cohort from FUSCC

\begin{tabular}{llll}
\hline Characteristic & $\begin{array}{l}\text { PDPN(+) } \\
\text { group } \\
\text { (N=28) }\end{array}$ & $\begin{array}{l}\text { PDPN(-) } \\
\text { group } \\
\text { (N=42) }\end{array}$ & P \\
\hline Gender & & & 0.56 \\
$\quad$ Male & $26(92.9)$ & $4 I(97.6)$ & \\
$\quad$ Female & $2(7.1)$ & I (2.4) & \\
Age (years) & $61.6 \pm 1.4$ & $61.8 \pm 1.3$ & 0.91 \\
Tumor largest dimension (cm) & $4.8 \pm 0.7$ & $4.3 \pm 0.6$ & 0.61 \\
Lymph node status & & & 0.05 \\
NO & $13(46.4)$ & $28(66.7)$ & \\
NI & $7(25.0)$ & $11(26.2)$ & \\
N2 & $8(28.6)$ & $3(7.1)$ & \\
AJCC stage & & & 0.30 \\
I & $8(28.6)$ & $18(42.9)$ & \\
II & $9(32.1)$ & $16(38.1)$ & \\
III & $10(35.7)$ & $7(16.7)$ & \\
IV & $1(3.6)$ & $1(2.4)$ & \\
\hline
\end{tabular}

Note: Data are shown as $\mathrm{n}(\%)$ or mean \pm SE.

Abbreviations: SqNSCLC, squamous non-small cell lung cancer; FUSCC, Fudan University Shanghai Cancer Center; PDPN, podoplanin; AJCC, American Joint Committee on Cancer. 
Table 2 Clinicopathological parameters of SqNSCLC patients in the validation cohort from TCGA

\begin{tabular}{|c|c|c|c|}
\hline Characteristic & $\begin{array}{l}\text { PDPN } \\
\text { mRNA high- } \\
\text { level group } \\
\end{array}$ & $\begin{array}{l}\text { PDPN } \\
\text { mRNA low- } \\
\text { level group }\end{array}$ & $p$ \\
\hline & $(N=I \mid 2)$ & $(N=|| 2)$ & \\
\hline Gender & & & 0.55 \\
\hline Male & $84(75.0)$ & $80(71.4)$ & \\
\hline Female & $28(25.0)$ & $32(28.6)$ & \\
\hline Age (years) & $66.1 \pm 8.6$ & $68.9 \pm 9.3$ & 0.02 \\
\hline Ethnicity & & & 0.20 \\
\hline White & $73(65.2)$ & $90(80.4)$ & \\
\hline Black or African American & $9(8.0)$ & $5(4.5)$ & \\
\hline Asian & $3(2.7)$ & I (0.9) & \\
\hline Unknown & $27(24.1)$ & $16(14.3)$ & \\
\hline AJCC stage & & & 1.00 \\
\hline I & $62(55.4)$ & $62(55.4)$ & \\
\hline II & $33(29.5)$ & $33(29.5)$ & \\
\hline III/IV & $17(15.2)$ & $17(15.2)$ & \\
\hline Lymph node status & & & 0.37 \\
\hline No & $79(70.5)$ & $73(65.2)$ & \\
\hline NI & $27(24.1)$ & $26(23.2)$ & \\
\hline N2 & $5(4.5)$ & $12(10.7)$ & \\
\hline N3 & I (0.9) & I (0.9) & \\
\hline $\mathrm{Nx}$ & - & - & \\
\hline Smoking & & & 0.72 \\
\hline Lifetime nonsmokers & $3(2.7)$ & $5(4.5)$ & \\
\hline Former smokers & $77(68.8)$ & 76 (67.9) & \\
\hline Current smokers & $30(26.8)$ & $27(24.1)$ & \\
\hline Unknown & $2(1.8)$ & $4(3.6)$ & \\
\hline
\end{tabular}

Note: Data are shown as $n(\%)$ or mean \pm SE.

Abbreviations: SqNSCLC, squamous non-small cell lung cancer; TCGA, The Cancer Genome Atlas; PDPN, podoplanin; AJCC, American Joint Committee on Cancer.

cohort, which consisted of consecutive patients with a diagnosis of SqNSCLC treated at FUSCC, a total of 70 patients who met the criteria were analyzed (Table 1 and Figure 1). To confirm the impact of PDPN on patient prognosis observed in the derivation group, a case-matched validation cohort was then identified with a total of 112 matched pairs of SqNSCLC (Table 2 and Figure 1).

For the derivation cohort of 70 patients, PDPN immunohistochemistry showed that $40 \%$ of the patients ( 28 cases) were PDPN positive (Figure 2). Cases with elevated PDPN expression had significantly increased pleural invasion ( $46.4 \%$ vs $9.5 \%, p=0.001)$, more LVI $(25.0 \%$ vs $9.5 \%$, $p=0.08$ ), and more lymph node involvement (53.6\% vs $33.3 \%, p=0.09$ ) (Figure 3). With a median follow-up of 53.4 months (95\% CI 38.0-68.9 months), marginally significantly shorter PFS was observed in the PDPN-positive group ( $p=0.07, \mathrm{HR}=2.15,95 \%$ CI $0.93-6.09$ ) (Figure 4a). The median PFS for the PDPN-positive group was 66.3 months
(95\% CI 39.6-92.9 months), while the median PFS for the PDPN-negative group was not reached. The estimated 5-year PFS rates were $54.7 \%$ and $72.3 \%$ for the PDPN-positive and PDPN-negative groups, respectively.

As in the case-matched validation cohort from TCGA data set, the median follow-up time was 34.6 months (95\% CI 28.9-40.2 months), with a total of 90 deaths. In these patients, OS was significantly different between PDPN mRNA dichotomized groups, with shorter OS observed in the high $P D P N$ mRNA group $(p=0.05, \mathrm{HR}=1.504,95 \% \mathrm{CI}$ 1.003-2.301) (Figure 4b). The median OS was 39.1 months (95\% CI 12.9-65.2 months) for the high PDPN mRNA group and 65.2 months (95\% CI 47.5-82.9 months) for the low $P D P N$ mRNA group. The estimated 5-year OS rates were $45.1 \%$ and $54.3 \%$ for the high $P D P N$ mRNA and low $P D P N$ mRNA groups, respectively. These clinical data from FUSCC and TCGA clearly indicate that SqNSCLC patients with an elevated expression of PDPN have worse clinicopathological features and poorer prognosis.

\section{PDPN showed only limited expression in normal human tissues}

An important advance in cancer treatment is the development of targeted therapies that attack a target expressed by tumor cells instead of normal tissues to avoid an off-target effect. ${ }^{25}$ Hence, we compared the protein expression of PDPN with other established targets ${ }^{7}$ that have been widely investigated to treat NSCLC using the HPM portal. The results showed that PDPN had no expression in several major adult organs, including cortex, spinal cord, heart, lung, kidney, gastrointestinal tract, and major immune cells, although PDPN had moderate to high expression in fetal brain and placenta (data not shown). Only mild PDPN expression was found in the adult liver, ovaries, testis, gallbladder, and urinary bladder, especially compared to the fact that popular established tumor targets, including EGFR, FGFR1, K-Ras, MET, and PIK3CA, had higher and broader expression in normal tissues (Figure 5). These results indicated that PDPN had high specificity as a potential target for SqNSCLC.

\section{Anti-PDPN NZ-I-IT showed specific and significant cytotoxicity in the human SqNSCLC cell line with elevated PDPN expression}

According to the NCI-60 cell line study, ${ }^{26}$ the H226 cell line (SqNSCLC cell line) showed a much higher expression (z-score 3.19) of PDPN mRNA than that of the negative 

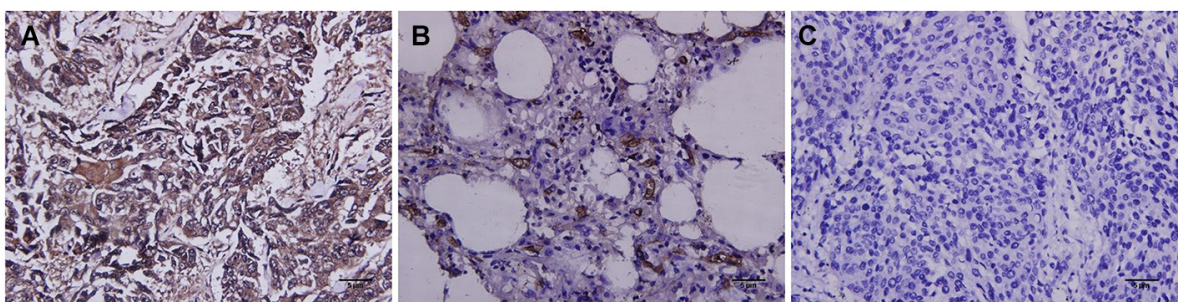

Figure 2 Immunohistochemical detection of PDPN in SqNSCLC patient samples: (A) PDPN-positive SqNSCLC section; (B) section displaying PDPN-negative staining in adjacent normal lung tissue and some positive staining in endothelial cells of lymphatic vessels; (C) PDPN-negative SqNSCLC section ( $\times 400$, bar=5 $\mu \mathrm{m})$.

Abbreviations: PDPN, podoplanin; SqNSCLC, squamous non-small cell lung cancer.

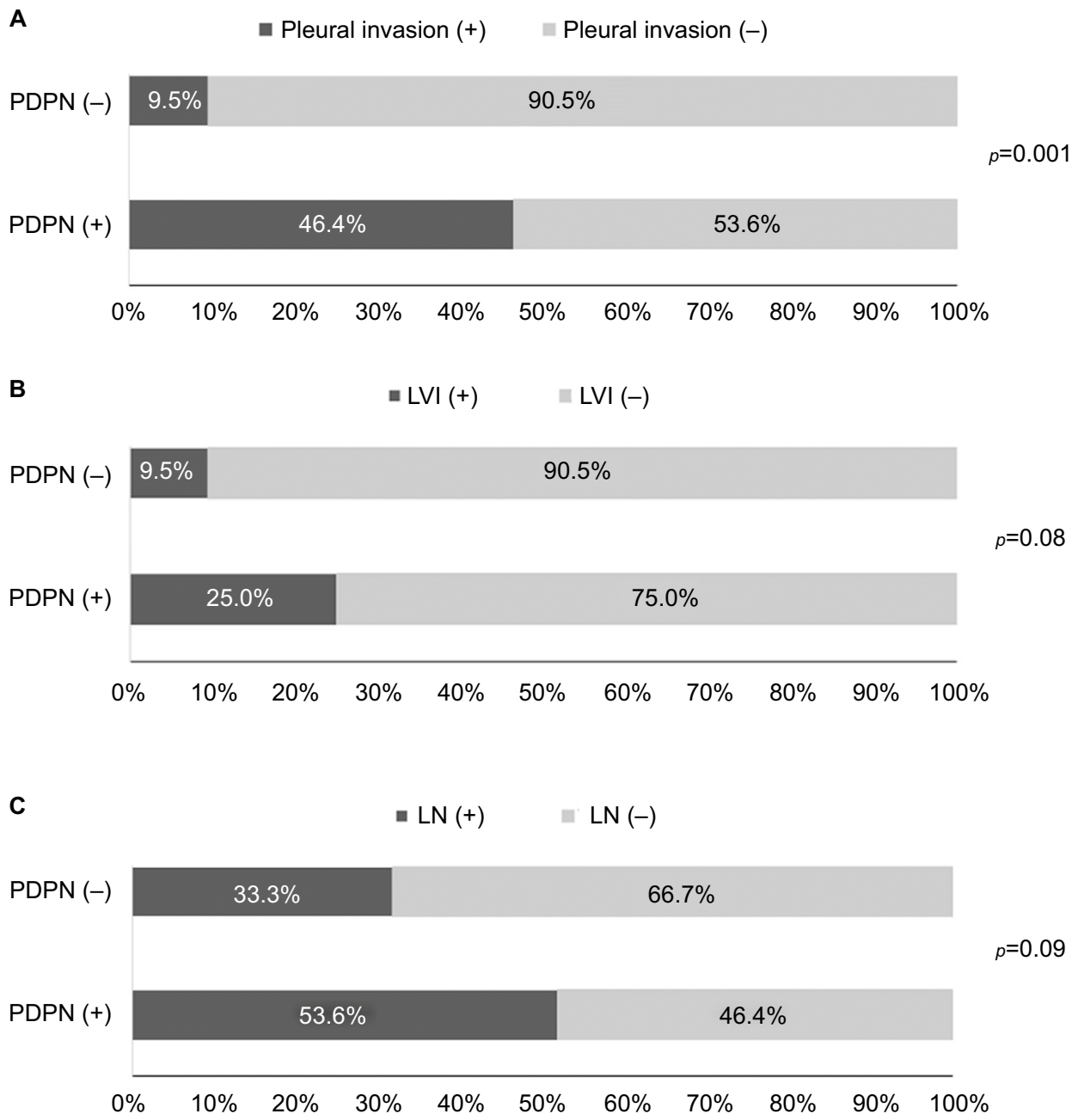

Figure 3 Pleural invasion, LVI, and LN involvement in SqNSCLC patients: (A) 9.5\% and 46.4\% of PDPN-negative and PDPN-positive SqNSCLC patients showed pleural invasion, respectively $(p=0.00 \mathrm{I})$; (B) $9.5 \%$ and $25 \%$ of PDPN-negative and PDPN-positive SqNSCLC patients showed LVI, respectively ( $p=0.08$ ); (C) $33.3 \%$ and $53.6 \%$ of PDPN-negative and PDPN-positive SqNSCLC patients showed LN involvement, respectively $(p=0.09)$.

Abbreviations: LVI, lymphovascular invasion; LN, lymph node; SqNSCLC, squamous non-small cell lung cancer; PDPN, podoplanin.

control, A549 cell line (z-score -0.45) (Figure 6a). This was confirmed by total PDPN protein expression using Western blot analysis. PDPN had a strong enrichment in the H226 cell line, while it was not detected in A549 cell line (Figure 6b). Surface PDPN expression was also much higher in the
H226 cell line than in the A549 cell line in flow cytometry analysis (Figure $6 \mathrm{c}$ and $6 \mathrm{~d}$ ). These experiments confirmed that the human SqNSCLC H226 cell line was a good model to investigate the efficacy of anti-PDPN therapy, whereas the A549 cell line could be used as the negative control cell line. 

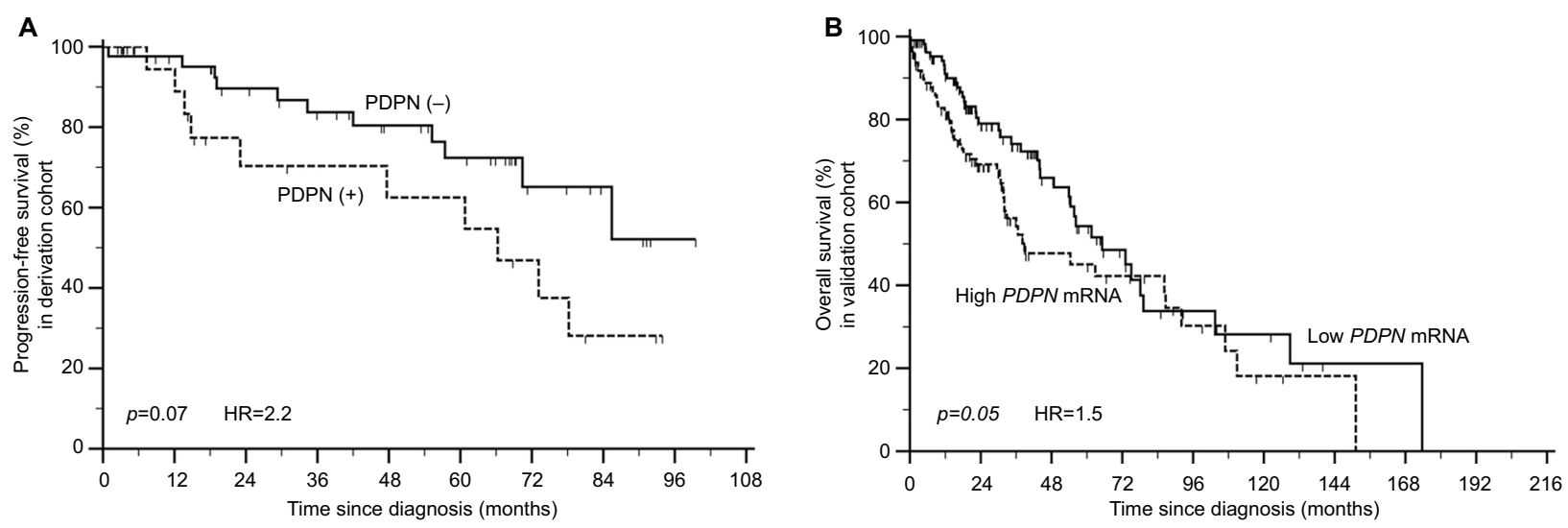

Figure 4 Association between PDPN expression and prognosis in SqNSCLC patients: (A) association between PDPN protein expression and progression-free survival in SqNSCLC patients in the derivation cohort ( $p=0.07)$; (B) association between PDPN mRNA expression and overall survival in case-matched SqNSCLC patients in the validation cohort $(p=0.05$, log-rank test, $\mathrm{HR}=1.504,95 \% \mathrm{Cl}$ I.003-2.30I).

Abbreviations: PDPN, podoplanin; SqNSCLC, squamous non-small cell lung cancer.

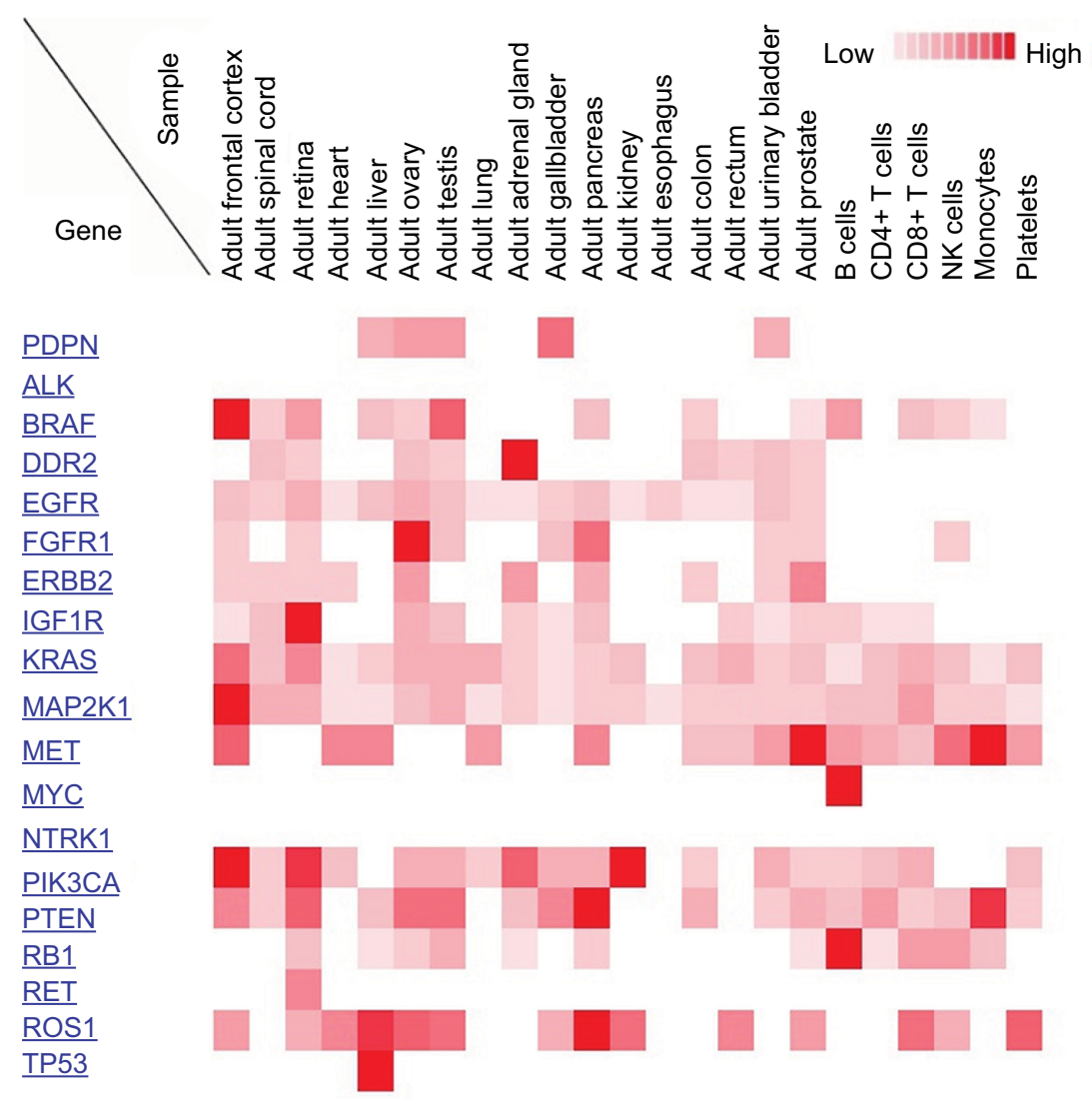

Figure 5 Expression of PDPN and other popular lung cancer targets in normal human tissues. Protein expression is depicted by a heat map (red indicates higher expression) in the Human Proteome Map portal.

Abbreviations: PDPN, podoplanin; ALK, anaplastic lymphoma kinase; BRAF, v-raf murine sarcoma viral oncogene homolog BI; DDR2, discoidin domain receptor 2; EGFR, epidermal growth factor receptor; FGFRI, fibroblast growth factor receptor I; ERBB2, v-erb-b2 erythroblastic leukemia viral oncogene homolog 2; IGFIR, insulin-like growth factor I receptor; K-Ras, Kirsten rat sarcoma viral oncogene homolog; MAP2KI, dual-specificity mitogen-activated protein kinase kinase I; MET, tyrosine-protein kinase Met; MYC, myelocytomatosis oncogene cellular homolog; NTRKI, neurotrophic tyrosine kinase receptor type I; PIK3CA, phosphatidylinositol-4,5-bisphosphate 3-kinase, catalytic subunit alpha; PTEN, phosphatase and tensin homolog deleted on chromosome 10; RBI, retinoblastoma protein; RET, rearranged during transfection proto-oncogene; ROSI, proto-oncogene tyrosine-protein kinase ROS; TP53, tumor protein p53. 
A

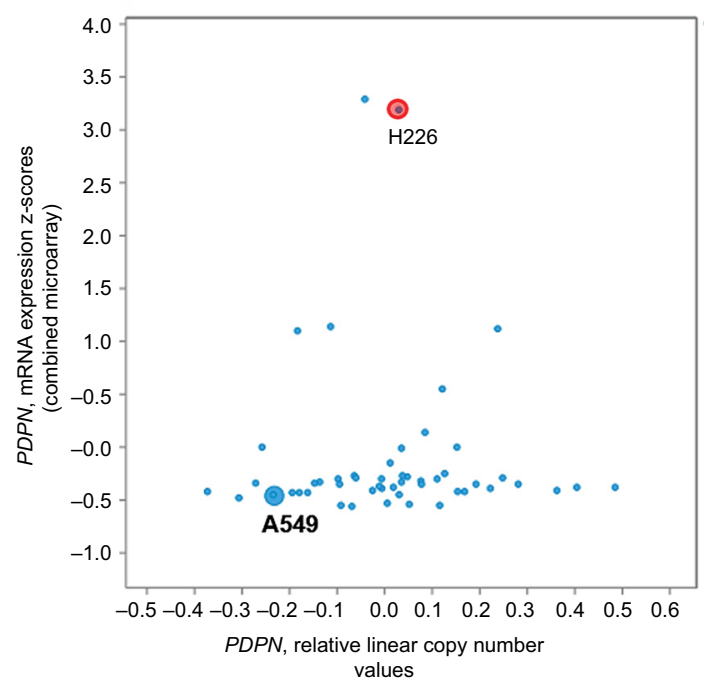

B

- Not mutated$$
\text { Cor }
$$
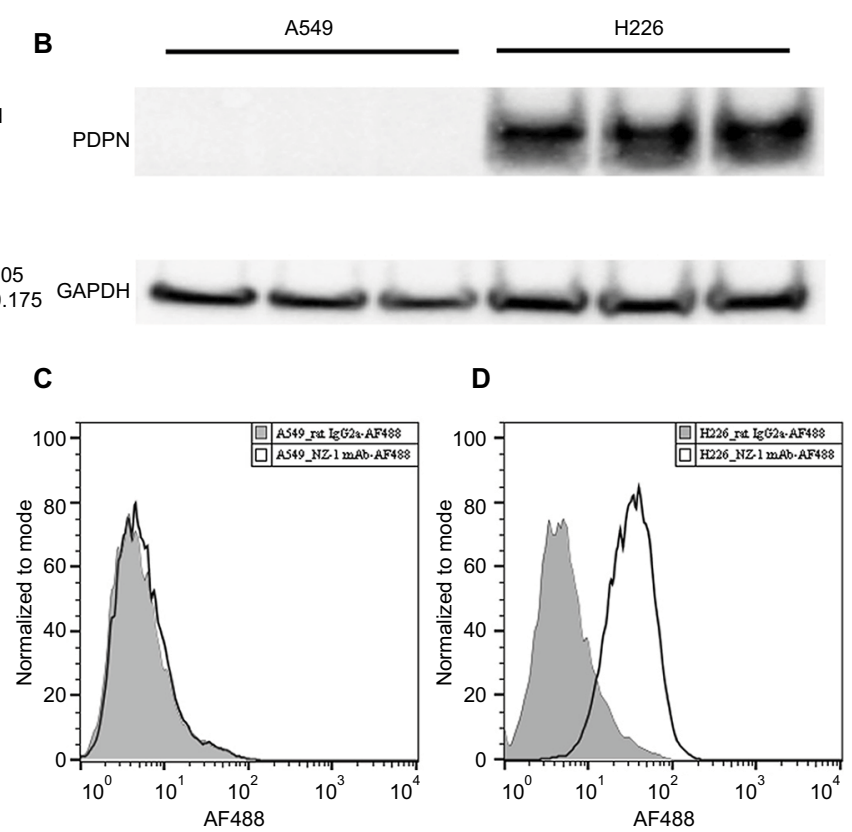

Figure 6 PDPN expression in A549 and H226 human NSCLC cell lines: (A) PDPN mRNA expression in A549 and H226 cell lines from the NCl-60 cell lines study; (B) Western blot analyses of PDPN expression in A549 and H226 cell lines. GAPDH was used as an internal reference. (C, D) Flow cytometry analyses of A549 and H226 cells to determine PDPN expression on their surface. Cell lines were stained with anti-PDPN-AF488 antibody (open black peaks) or an isotype control antibody (filled gray peaks). Abbreviations: PDPN, podoplanin; NSCLC, non-small cell lung cancer; GAPDH, glyceraldehyde 3-phosphate dehydrogenase.

Based on this model, we then examined the effect of NZ-1-IT on A549 and H226 human NSCLC cell lines. The cytotoxicity of NZ-1-IT was determined by the WST-1 assay. We found that NZ-1-IT was hardly able to kill PDPN-negative A549 cells $\left(\mathrm{IC}_{50}>1000 \mathrm{ng} / \mathrm{mL}\right.$ ) (Figure 7a), whereas PDPNpositive $\mathrm{H} 226$ cells were quite sensitive to NZ-1-IT treatment, with $\mathrm{IC}_{50}$ as low as $1.205 \mathrm{ng} / \mathrm{mL}$ (Figure $7 \mathrm{~b}$ ). These in vitro cytotoxicity results suggested that PDPN could be a robust target for antitumor therapy and NZ-1-IT specifically targets PDPN-positive SqNSCLC cells.

\section{Anti-PDPN NZ-I-IT significantly delayed tumor growth in SqNSCLC xenografts with elevated expression of PDPN}

Considering the promising in vitro results, the efficacy of anti-PDPN therapy was further investigated in nude mice bearing A549 or H226 subcutaneous xenograft tumors. Subcutaneous tumors were treated by NZ-1-IT or vehicle control. Similar to the in vitro result, anti-PDPN NZ-1-IT therapy failed to show any antitumor benefit in PDPN-negative, A549-bearing mice (Figure 7c). However, the tumor growth was significantly delayed in the NZ-1-IT-treated group in PDPN-positive H226-bearing mice $(p<0.05)$ (Figure $7 \mathrm{~d}$ ). No toxicity-related adverse effects were observed. These in vivo mouse xenograft NSCLC models further confirmed that anti-PDPN therapy could be a promising targeted therapy to treat SqNSCLC tumors.

\section{Discussion}

This study revealed for the first time that elevated tumor PDPN expression on both the mRNA level and the protein level has a significant impact on clinicopathological features (pleural invasion, LVI, and lymph node involvement) along with patient survival in SqNSCLC by analyzing two separate patient cohorts. We also found that PDPN distinctly had no or mild expression in normal tissue, compared to other commonly used therapeutic targets. We further demonstrated that targeting PDPN significantly impeded tumor cell survival in vitro using an anti-PDPN immunotoxin, NZ-1-IT. Moreover, in vivo mouse models confirmed that anti-PDPN therapy significantly delayed the growth of elevated PDPN-expressing SqNSCLC xenografts, with no adverse effects observed. Taken together, these data have provided strong evidence to support that PDPN is a robust target with high potential against SqNSCLC and suggest that anti-PDPN therapy could be a promising method for SqNSCLC treatment.

PDPN has been reported to induce invasion and migration of cancer cells via the downregulation of small Rho family GTPases, providing an alternative tumor cell invasion 

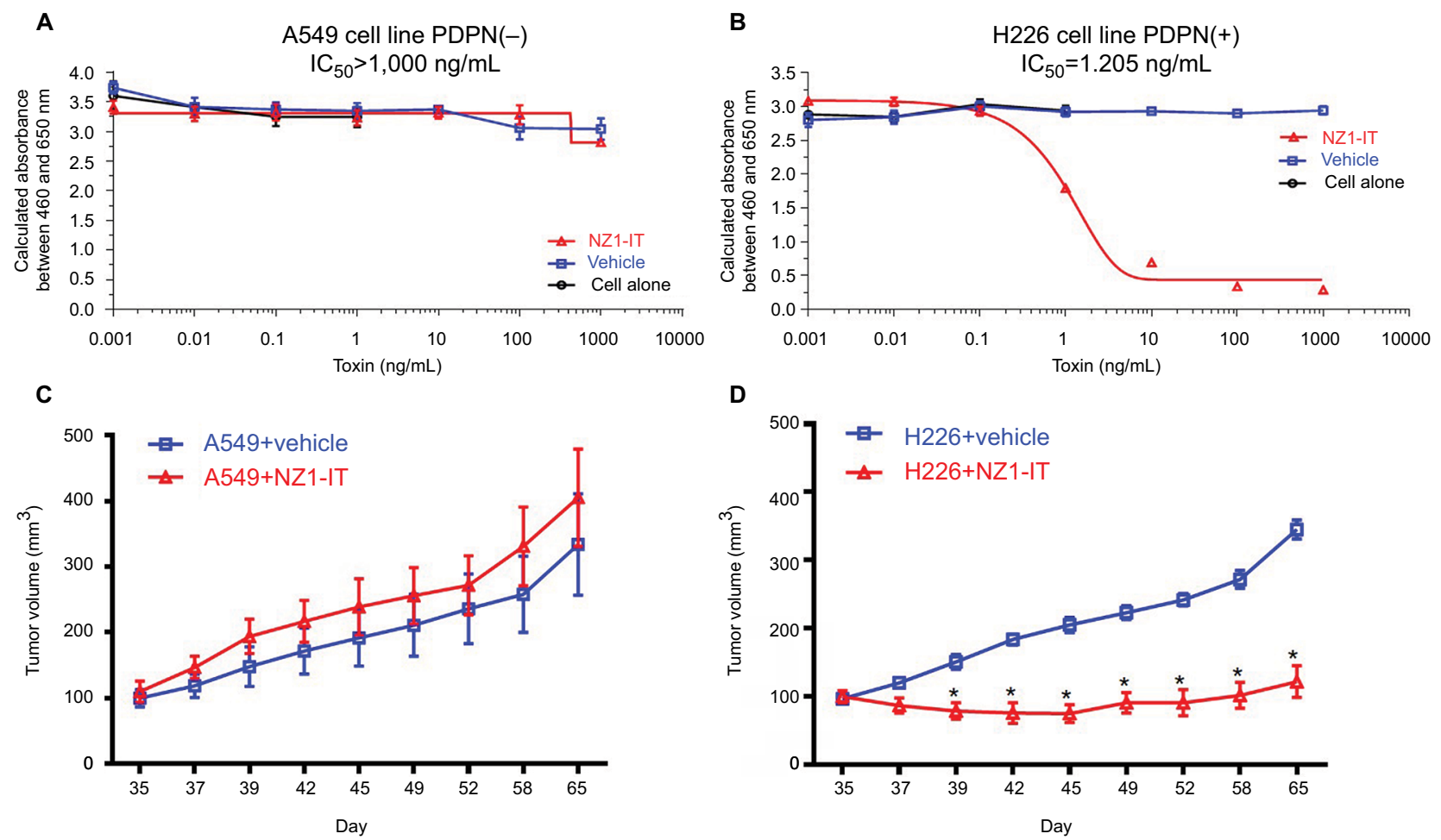

D

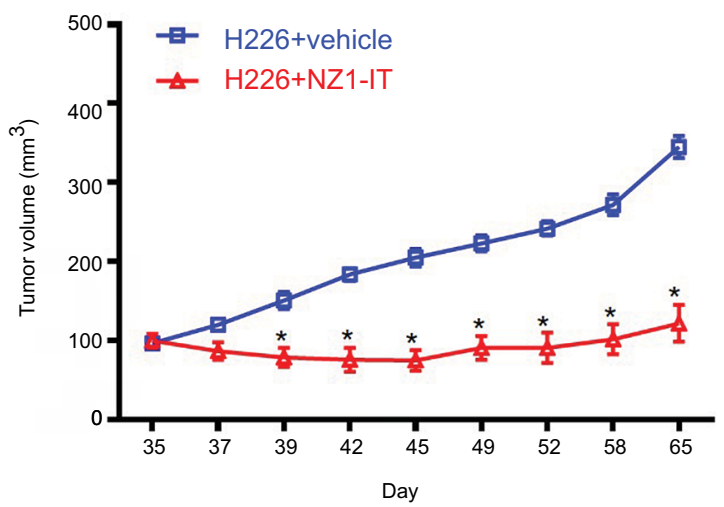

Figure 7 Preclinical therapeutic efficacy of anti-PDPN NZ-I-IT in in vitro and in vivo SqNSCLC models. In vitro cytotoxicity of NZ-I-IT against (A) non-squamous A549 and (B) squamous $\mathrm{H} 226$ cell lines was determined by WST-I assay. NZ-I IT was serially diluted to achieve final concentrations of $0.001,0.01,0.1, I, 10,100$, and I000 ng/ $\mathrm{mL}$ in $0.2 \%$ human serum albumin-PBS. $\Delta$, NZ-I-IT treated group; $\square$, vehicle group; $\circ$, cell-alone group. Effect of NZ-I-IT against A549 and H226 subcutaneous tumors was examined in xenograft mouse NSCLC models. Female athymic nude mice bearing A549 tumors (C) or H226 tumors (D) were randomized into two groups: vehicle ( $\square$ ) and NZ-I-IT $(\Delta)$. Once the tumors reached an average volume of $100 \mathrm{~mm}^{3}$ on day 35 , the mice were treated by intratumoral injections every other day with a total of three $300 \mu \mathrm{g} / \mathrm{kg}$ doses of NZ-I-IT or vehicle on days 35,37 , and 39 after the tumor implantation. $\left({ }^{*} p<0.05\right)$.

Abbreviations: PDPN, podoplanin; NZ-I-IT, NZ-I-immunotoxin; SqNSCLC, squamous non-small cell lung cancer; NSCLC, non-small cell lung cancer.

pathway in the absence of EMT processes (including cadherin switching). ${ }^{27}$ It can also increase the adhesion and spreading of cancer cells on the fibronectin protein of the extracellular matrix to provide a microenvironment for further tumor cell migration and invasion, even in the presence of E-cadherin. ${ }^{12,27}$ PDPN is also related to ezrin, which mediates the process of tumor metastasis. ${ }^{28}$ These mechanisms may explain our finding that patients with elevated PDPN expression showed a significantly increasing rate of pleural invasion, known as pathological invasiveness in SqNSCLC. In addition, PDPN has been reported to play a critical role in promoting the metastasis of tumor cells through lymphovascular vessels to invade drainage lymph nodes in other cancers..$^{29,30}$ Although our results showed only a marginal increase in LVI and lymph node involvement in those SqNSCLC cases with elevated PDPN expression, they still strongly suggest that there may be a causal relation between PDPN level and lymph node metastasis in SqNSCLC, which needs to be further examined in more patients. Overall, as the current study revealed many unique clinicopathological features of SqNSCLC, the above factors may at least partly explain the worse prognosis in those patients with elevated PDPN expression.

During the last decade, research advances, including the development of molecular cancer genetics, targeted therapies, and large-scale global databases, have significantly shifted the paradigm of cancer treatment from solely conventional therapies to integrating targeted, precise, and personalized therapies. ${ }^{7,31,32}$ As mentioned earlier, it is crucial for these targeted therapeutic methods to recognize specifically unique molecular abnormalities in squamous cell lung cancer instead of the normal tissues. Hence, a significant distinct expressed molecular target between tumor and normal tissue is ideal for these therapies. In our derivation cohort, we found an elevated expression of PDPN in as many as $40 \%$ of SqNSCLC cases, while PDPN showed no or only mild expression in major adult human organs compared with many other popular targets currently used in the clinic, although data from PDPN-deficient mice showed that PDPN is crucial during the development of the heart, lung, and lymphatic 
systems. ${ }^{33,34}$ In spite of the result that the HPM database did not show PDPN expression in adult lung, a previous study reported that $P D P N$ transcripts had a low-level expression in human lung tissue. ${ }^{35}$ Furthermore, with the development of modern techniques, antitumor agents can be locoregionally delivered into the lung tumor site without any serious adverse events in patients. ${ }^{17}$ Immunotoxin therapy through locoregionally intratumoral delivery has already been used to successfully treat patients with brain cancer, to specifically target tumor cells and protect the normal tissue that may also express similar targets. ${ }^{17}$ Overall, these findings suggested that an anti-PDPN therapy is able to target tumor cells with limited off-target effects.

In the current study, PDPN protein level in the derivation cohort was interrogated via immunohistochemistry in 70 SqNSCLC patients. In the validation cohort from TCGA data set, PDPN expression was examined on the mRNA level, with more than 100 matched pairs of cases, matched based on AJCC cancer stage. Given the relatively small scale of the FUSCC cohort and to avoid bias from the few events of death, the end point for this derivation cohort was defined by PFS instead of OS for analyzing the association of PDPN and patient prognosis. On the other hand, OS was used for the validation cohort because TCGA data set contains complete survival data with an adequate follow-up but often lacks disease progression details. Despite these caveats, a strong relationship between elevated PDPN expression and poor prognosis is suggested in survival comparisons between the two different populations.

As a subsequent investigation on the overall effect of PDPN on SqNSCLC treatment, including its obviously distinct expression in lung tumors as well as its association with poor patient prognosis, the promising findings from in vitro and in vivo experiments are believed to be the first evidence to show the therapeutic effect of anti-PDPN therapy using a targeted immunotoxin, NZ-1-IT, for SqNSCLC treatment at the preclinical level, with robust potential. Furthermore, the antitumor immunotoxin therapy can induce a secondary antitumor immunity in addition to its specific and direct killing via the toxin-induced protein synthesis inhibition. Leshem et $\mathrm{al}^{36}$ reported that immunotoxin can synergize with an immune checkpoint inhibitor, leading to a higher rate of complete regression in mouse breast cancer models owing to an increase of tumor-infiltrating $\mathrm{CD} 8^{+} \mathrm{T}$ cells, indicating that immunotoxin can promote the development of anti-tumor immunity to achieve long-term tumor elimination. Currently, two anti-PD-1 immune checkpoint inhibitors have been approved by the US Food and Drug Administration for NSCLC treatment, and are potential candidates to be combined with anti-PDPN immunotoxins to treat PDPN-positive SqNSCLC in the future.

\section{Conclusion}

Our results clearly demonstrate that there is a significantly distinct expression of PDPN in SqNSCLC and normal tissue, and its elevated expression is associated with worse clinicopathological characteristics along with poor prognosis in SqNSCLC patients. With the promising therapeutic results from both in vitro and in vivo studies, anti-PDPN therapy can thus be developed as a robust potential targeted therapy for SqNSCLC treatment in the future.

\section{Abbreviations}

NSCLC: non-small cell lung cancer

SqNSCLC: squamous non-small cell lung cancer

PDPN: podoplanin

cAMP: cyclic adenosine monophosphate

CLEC2: C-type lectin-like receptor 2

ERM: ezrin-radixin-moesin

EMT: epithelial-mesenchymal transition

NZ-1-IT: NZ-1-immunotoxin

scFv: single-chain variable-region antibody fragment

mAb: monoclonal antibody

ADC: antibody-drug conjugate

LVI: lymphovascular invasion

FUSCC: Fudan University Shanghai Cancer Center

IRB: institutional review board

AJCC: American Joint Committee on Cancer

EMR: electronic medical record

TCGA: The Cancer Genome Atlas

HPM: Human Proteome Map

ATCC: American Type Culture Collection

FBS: fetal bovine serum

GAPDH: glyceraldehyde 3-phosphate dehydrogenase

HSA: human serum albumin

OS: overall survival

PFS: progression-free survival

EGFR: epidermal growth factor receptor

FGFR1: fibroblast growth factor receptor 1

K-Ras: Kras Kirsten rat sarcoma viral oncogene homolog

MET: tyrosine-protein kinase Met

PIK3CA: phosphatidylinositol-4,5-bisphosphate 3-kinase, catalytic subunit alpha

PD-1: programmed cell death protein 1

\section{Acknowledgments}

We would like to thank Jenna Lewis for editing our manuscript. 
The study was supported by grants from the National Natural Science Foundation of China (No. 81401896, to L Xie), and from Shanghai Municipal Human Resource Bureau and Shanghai Science and Technology Committee (No. 14PJ1402000, to L Xie).

\section{Disclosure}

DD Bigner owns stock in Istari Oncology and is a consultant to Genetron Health. DD Bigner and X Bao share a patent on combination therapy of immunotoxin and checkpoint inhibitor. The other authors report no conflicts of interest in this work.

\section{References}

1. Chen W, Zheng R, Baade PD, et al. Cancer statistics in China, 2015. CA Cancer J Clin. 2016;66(2):115-132.

2. Ettinger DS, Wood DE, Aisner DL, et al. Non-Small Cell Lung Cancer, Version 5.2017, NCCN Clinical Practice Guidelines in Oncology. J Natl Compr Canc Netw. 2017;15(4):504-535.

3. Derman BA, Mileham KF, Bonomi PD, Batus M, Fidler MJ. Treatment of advanced squamous cell carcinoma of the lung: a review. Transl Lung Cancer Res. 2015;4(5):524-532.

4. Molina JR, Yang P, Cassivi SD, Schild SE, Adjei AA. Non-small cell lung cancer: epidemiology, risk factors, treatment, and survivorship. Mayo Clin Proc. 2008;83(5):584-594.

5. Alberg AJ, Brock MV, Samet JM. Epidemiology of lung cancer: looking to the future. J Clin Oncol. 2005;23(14):3175-3185.

6. Chen Z, Fillmore CM, Hammerman PS, Kim CF, Wong KK. Non-smallcell lung cancers: a heterogeneous set of diseases. Nat Rev Cancer. 2014;14(8):535-546.

7. Tan W-L, Jain A, Takano A, et al. Novel therapeutic targets on the horizon for lung cancer. Lancet Oncol. 2016;17(8):e347-e362.

8. Scott AM, Wolchok JD, Old LJ. Antibody therapy of cancer. Nat Rev Cancer. 2012;12(4):278-287.

9. Gandara DR, Hammerman PS, Sos ML, Lara PN Jr, Hirsch FR. Squamous cell lung cancer: from tumor genomics to cancer therapeutics. Clin Cancer Res. 2015;21(10):2236-2243.

10. Fujita N, Takagi S. The impact of Aggrus/podoplanin on platelet aggregation and tumour metastasis. J Biochem. 2012;152(5):407-413.

11. Martin-Villar E, Megias D, Castel S, Yurrita MM, Vilaro S, Quintanilla M. Podoplanin binds ERM proteins to activate RhoA and promote epithelial-mesenchymal transition. J Cell Sci. 2006;119(Pt 21):4541-4553.

12. Wicki A, Christofori G. The potential role of podoplanin in tumour invasion. Br J Cancer. 2007;96(1):1-5.

13. Kunita A, Kashima TG, Morishita Y, et al. The platelet aggregationinducing factor aggrus/podoplanin promotes pulmonary metastasis. Am J Pathol. 2007;170(4):1337-1347.

14. Chandramohan V, Bao X, Kato Kaneko M, et al. Recombinant antipodoplanin (NZ-1) immunotoxin for the treatment of malignant brain tumors. Int J Cancer. 2013;132(10):2339-2348.

15. Chandramohan V, Bao X, Keir ST, et al. Construction of an immunotoxin, D2C7-(scdsFv)-PE38KDEL, targeting EGFRwt and EGFRvIII for brain tumor therapy. Clin Cancer Res. 2013;19(17):4717-4727.

16. Bao X, Chandramohan V, Keir ST, et al. Antitumor efficacy of D2C7(scdsFv)-PE38KDEL, a novel immunotoxin targeting EGFRwt and EGFRvIII, by convection-enhanced delivery in orthotopic brain tumor mouse models. J Immunother Cancer. 2013;1(Suppl 1):P126.

17. Xie LY, Piao HL, Fan M, et al. Immunotoxin therapy for lung cancer. Chin Med J (Engl). 2017;130(5):607-612.

18. Hassan R, Alewine C, Pastan I. New life for immunotoxin cancer therapy. Clin Cancer Res. 2016;22(5):1055-1058.
19. Gao J, Aksoy BA, Dogrusoz U, et al. Integrative analysis of complex cancer genomics and clinical profiles using the cBioPortal. Sci Signal. 2013;6(269):pl1.

20. Cerami E, Gao J, Dogrusoz U, et al. The cBio cancer genomics portal: an open platform for exploring multidimensional cancer genomics data. Cancer Discov. 2012;2(5):401-404.

21. Kim MS, Pinto SM, Getnet D, et al. A draft map of the human proteome. Nature. 2014;509(7502):575-581.

22. Kaplan EL, Meier P. Nonparametric estimation from incomplete observations. J Am Stat Assoc. 1958;53(282):457-481.

23. Mantel N. Evaluation of survival data and two new rank order statistics arising in its consideration. Cancer Chemother Rep. 1966;50(3): $163-170$.

24. Cox DR. Regression models and life-tables. J R Stat Soc B. 1972;34(2): $187-220$.

25. Sawyers C. Targeted cancer therapy. Nature. 2004;432(7015):294-297.

26. Abaan OD, Polley EC, Davis SR, et al. The exomes of the NCI-60 panel: a genomic resource for cancer biology and systems pharmacology. Cancer Res. 2013;73(14):4372-4382.

27. Wicki A, Lehembre F, Wick N, Hantusch B, Kerjaschki D, Christofori G. Tumor invasion in the absence of epithelial-mesenchymal transition: podoplanin-mediated remodeling of the actin cytoskeleton. Cancer Cell. 2006;9(4):261-272.

28. Yu Y, Khan J, Khanna C, Helman L, Meltzer PS, Merlino G. Expression profiling identifies the cytoskeletal organizer ezrin and the developmental homeoprotein Six-1 as key metastatic regulators. Nat Med. 2004;10(2):175-181.

29. Rodrigo JP, Garcia-Carracedo D, Gonzalez MV, Mancebo G, Fresno MF, Garcia-Pedrero J. Podoplanin expression in the development and progression of laryngeal squamous cell carcinomas. Mol Cancer. 2010;9:48.

30. Yuan P, Temam S, El-Naggar A, et al. Overexpression of podoplanin in oral cancer and its association with poor clinical outcome. Cancer. 2006;107(3):563-569.

31. Hirsch FR, Scagliotti GV, Mulshine JL, et al. Lung cancer: current therapies and new targeted treatments. Lancet. 2017;389(10066):299-311.

32. Sculier JP, Berghmans T, Meert AP. Advances in target therapy in lung cancer. Eur Respir Rev. 2015;24(135):23-29.

33. Ramirez MI, Millien G, Hinds A, Cao Y, Seldin DC, Williams MC. T1alpha, a lung type I cell differentiation gene, is required for normal lung cell proliferation and alveolus formation at birth. Dev Biol. 2003;256(1):61-72.

34. Mahtab EA, Wijffels MC, Van Den Akker NM, et al. Cardiac malformations and myocardial abnormalities in podoplanin knockout mouse embryos: correlation with abnormal epicardial development. Dev Dyn. 2008;237(3):847-857.

35. Martin-Villar E, Scholl FG, Gamallo C, et al. Characterization of human PA2.26 antigen (T1alpha-2, podoplanin), a small membrane mucin induced in oral squamous cell carcinomas. Int $J$ Cancer. 2005;113(6):899-910.

36. Leshem Y, O’Brien J, Liu X, et al. Combining local immunotoxins targeting mesothelin with CTLA-4 blockade synergistically eradicates murine cancer by promoting anticancer immunity. Cancer Immunol Res. 2017;5(8):685-694.

37. World Health Organization. Cancer. February 2018. Available from: http://www.who.int/mediacentre/factsheets/fs297/en/. Accessed October 24, 2017.

38. World Health Organization. Tobacco in China. Available from: http:// www.wpro.who.int/china/mediacentre/factsheets/tobacco/en/. Accessed November 19, 2017.

39. cBioPortal for Cancer Genetics. Available from: http://www.cbioportal. org. Accessed September 15, 2017.

40. Human Proteome Map. Available from: http://humanproteomemap.org/. Accessed June 6, 2017.

41. American Type Culture Collection. Available from: http://www.atcc. org 


\section{Publish your work in this journal}

Cancer Management and Research is an international, peer-reviewed open access journal focusing on cancer research and the optimal use of preventative and integrated treatment interventions to achieve improved outcomes, enhanced survival and quality of life for the cancer patient. The manuscript management system is completely online and includes a very quick and fair peer-review system, which is all easy to use. Visit http://www.dovepress.com/testimonials.php to read real quotes from published authors. 